\title{
Medición de la participación en niños y adolescentes con y sin discapacidad: una revisión sistemática
}

\author{
Measuring participation in children and adolescents with and without disabilities: \\ a systematic review
}

\section{Paula Nahuelhual $^{\mathrm{a}}$, Carolina Giaconi ${ }^{\mathrm{b}}$, M. Alejandra Machuca ${ }^{\mathrm{b}}$}

aDirección de Investigación y Desarrollo. Teletón Chile

bVoluntariado Teletón Chile

Recibido el 4 de abril de 2017; aceptado el 2 de agosto de 2017

\section{Resumen}

Introducción: De acuerdo a la Clasificación Internacional del Funcionamiento de la Discapacidad y la Salud (CIF), la participación se define como "el involucramiento del sujeto en situaciones de la vida” y es un aspecto fundamental en el desarrollo de los niños, niñas y adolescentes. En el caso de niños(as) con discapacidad, permite una mejor comprensión del posible impacto de las deficiencias en la vida cotidiana. Objetivo: Evaluar las escalas de medición de la participación en niños, niñas y adolescentes con y sin discapacidad. Método: Revisión sistemática. Se incluyeron estudios de validación de instrumentos de evaluación de la participación en niños(as) y adolescentes con y sin discapacidad sin restricción de lenguaje. La búsqueda se realizó en Pubmed, EMBASE, CINAHL, The Cochrane Library, Biblioteca Virtual de la Salud, Opengrey y Google Scholar. Los datos fueron extraídos y analizados en Microsoft Excel. Registro de protocolo PROSPERO 2015:CRD42015020644. Resultados: De los 1.689 artículos que arrojó la búsqueda electrónica, se identificaron 9 escalas de evaluación de la participación de niños(as) y/o adolescentes con y sin discapacidad. Diversidad en las dimensiones, extensión y aplicación (padres y niños(as)). El número de pacientes incorporados en las validaciones originales es variable, al igual que el porcentaje de niños y/o adolescentes con discapacidad incluidos en los estudios de validación. Conclusiones: Existe gran variabilidad en las propiedades psicométricas y características de las escalas incluidas en esta revisión, principalmente debido a que el constructo de participación difiere de acuerdo a la cultura, por lo que las escalas seleccionadas requieren de adaptaciones transculturales para su uso.

\section{Abstract}

Introduction: According to the International Classification of Functioning, Disability and Health (ICF), participation is defined as "the involvement of the subject in situations of life" and is fundamental in the development of children and adolescents. In case of children with disabilities,
Palabras clave: Discapacidad, participación, niños, adolescentes

Keywords: Disabilities, participation, children, adolescents 
participation allows a better understanding of the possible impact of deficiencies in daily life. Objective: To evaluate measurement scales of participation in children and adolescents with and without disabilities. Method: Systematic review. Validation studies of measurement scales of participation in children and adolescents with and without disabilities without language restriction were included. The search was performed in Pubmed, EMBASE, CINAHL, The Cochrane Library, Health Virtual Library, Opengrey and Google Scholar. The data were extracted and analyzed in Microsoft Excel. Protocol Register PROSPERO 2015: CRD42015020644. Results: 1689 articles were collected through electronic search, 9 scales were selected for analysis. Diversity in size and application between the scales selected was found. The number of patients included in the original validations was variable, as the percentage of children and/or adolescents with disabilities included in the validation studies. Conclusions: There is great variability in the psychometric properties and characteristics of the scales included in this review, mainly for which the participation construct differs according to culture, so the selected scales require transcultural adaptations for their use.

\section{Introducción}

La Clasificación Internacional del Funcionamiento de la Discapacidad y la Salud (CIF), propuesta por la OMS en $2001^{1}$, propone un marco general para comprender la salud y la discapacidad desde la perspectiva del cuerpo y la perspectiva del individuo y la sociedad ${ }^{2}$. La CIF unifica los distintos conceptos de participación que se manejaban a la fecha ${ }^{3}$ y define la participación como el "involucramiento del sujeto en situaciones de la vida" comprendiéndose ésta como el resultado de la interacción de los individuos con su entorno físico, social y actitudinal ${ }^{1}$, y se convierte en una fuente para una mejor comprensión del posible impacto de las deficiencias en la vida de una persona individual ${ }^{4}$. Tras la publicación de la CIF, la comprensión de la discapacidad se torna multidimensional, dentro de la cual la participación se convierte en pilar fundamental para la comprensión de ésta y para el desarrollo de distintas alternativas que la incorporen como uno de los objetivos fundamentales a la hora de pensar en la rehabilitación de personas en situación de discapacidad.

La importancia de la participación en la población infantil y juvenil, radica en que es en este tipo de espacios y actividades de ocio y recreo, donde los niños, niñas y adolescentes desarrollan habilidades y competencias, alcanzan salud física y emocional, aprenden a relacionarse positivamente con otros, establecen amistades, expresan creatividad, desarrollan su propia identidad y construyen un significado y un propósito en la $v_{i d a}, 6,7,8$, además les permite desarrollar la motivación interna y efectuar actividades de elección propia ${ }^{9}$. La participación en diversas actividades de ocio y tiempo libre se ha convertido para diversos especialistas en desarrollo infantil y juvenil, en un indicador de funcionalidad y salud, ya que se correlaciona directamente con la calidad de vida y bienestar físico y emocional ${ }^{9,10,11}$.

En el caso de los(as) niños(as) con discapacidad, la participación les permite explorar su potencial social, intelectual, emocional, comunicativo y físico, siendo un importante predictor de su futura satisfacción con la vida ${ }^{5,12,13}$. Se han identificado distintos factores relacionados con la participación en estos niños, entre ellos, se ha reconocido que mayores niveles de función física, cognitiva y comunicativa, se asocian con mayor intensidad de la participación ${ }^{5}$. Desde la publicación de la CIF, la participación se ha convertido en uno de las áreas que mayor relevancia ha tomado, ya que se considera fundamental dentro del concepto de inclusión de estos niños y niñas ${ }^{11,14}$.

Se ha demostrado que la participación es distinta dependiendo de los contextos socio-culturales en que se desenvuelvan los niños y adolescentes y las variables como país de residencia, factores ambientales son factores predictores de la variabilidad de la participación en niños(as) con y sin discapacidad ${ }^{15}$. Esta dependencia contextual representa un desafío para establecer objetivos de rehabilitación relacionados con mejorar la participación en niño/adolescente con discapacidad.

La medición de la participación es un factor clave para identificar las distintas realidades que viven los (las) niños(as) en los distintos contextos y proponer las mejores intervenciones para equiparar las posibilidades de participación con sus pares que no están en situación de discapacidad ${ }^{16,17}$.

Existen diversas escalas que miden distintos ámbitos de la participación ${ }^{18,19,20,21,22}$, a través del autoreporte de los niños, niñas y adolescentes y/o a través del reporte de sus padres y/o cuidadores principales. Revisiones sistemáticas previas han identificado escalas aplicables a poblaciones con patologías particulares, como por ejemplo, parálisis cerebral ${ }^{23} \mathrm{o}$ a grupos particulares de discapacidades ${ }^{24,25,26}$. Sin embargo, no existen revisiones sistemáticas que recomienden las escalas que puedan aplicarse simultáneamente a niños(as) y adolescentes con y sin discapacidad. Disponer de estas escalas o instrumentos, permitiría comparar las brechas con niños(as) sin discapacidad, realizar una mejor derivación a los distintos programas e intervenciones que tengan por objetivo el mejoramiento de la parti- 
cipación infantil y juvenil, además, permitiría contar con mayor información para tomar decisiones clínicas que afecten este ámbito, entendiendo que en el proceso del desarrollo evolutivo, la posibilidad de participar en espacios informales, recreativos y de vinculación con pares genera aprendizajes que son significativos y trascendentales para el desarrollo de los niños, niñas y adolescente.

Las preguntas que pretende responder la presente revisión sistemática son: ¿Cuáles son los instrumentos disponibles para medir la participación de niños y adolescentes con y sin discapacidad? y ¿Cuáles son las propiedades psicométricas (confiabilidad y validez) de los instrumentos utilizados para medir la participación de niños(as) y adolescentes con y sin discapacidad? Con el objetivo de evaluar las escalas de medición de la participación en niños, niñas y adolescentes con y sin discapacidad disponibles actualmente en la literatura y de esta forma contar con una visión general respecto de las evaluaciones que se utilizan para estas mediciones.

\section{Método}

La presente revisión sistemática se realizó siguiendo las recomendaciones de la Colaboración Cochrane ${ }^{27}$ y la pauta para revisiones sistemáticas PRISMA ${ }^{28}$, y su protocolo fue registrado en PROSPERO, con el número de registro PROSPERO 2015:CRD42015020644 ${ }^{29}$.

Se incorporaron estudios de corte transversal y cohortes cuyo objetivo primario fuera la validación de instrumentos de participación en niños, niñas y adolescentes con y sin discapacidad, publicados a partir del año 2001 (año de publicación de la CIF). El criterio para identificar que los instrumentos fueran aplicables

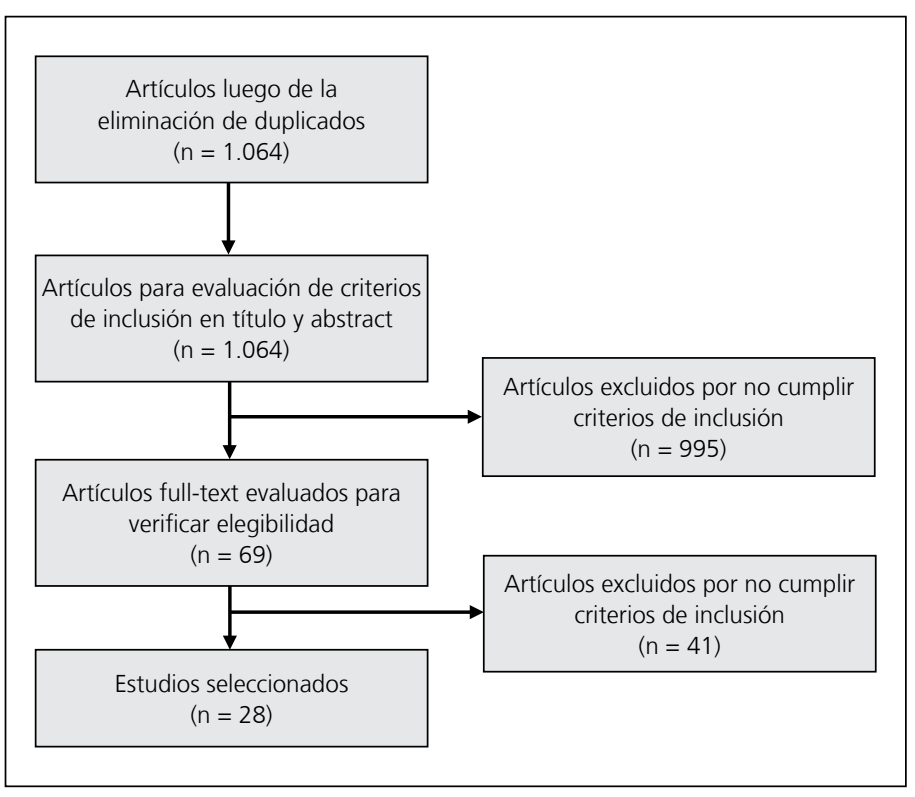

Figura 1. Proceso de selección de escalas de medición. a niños(as)/adolescentes con y sin discapacidad fue que los autores lo declararan como tal o que en el en el proceso de validación de los instrumentos hubieran incluido grupos con y sin discapacidad.

Como resultados primarios se consideraron las propiedades psicométricas de los instrumentos incluidos y como resultados secundarios sus características de aplicabilidad ${ }^{30}$.

La búsqueda sistemática se realizó en las siguientes bases de datos biomédicas: Pubmed, EMBASE, CINAHL, The Cochrane Library, Biblioteca Virtual de la Salud, Opengrey y Google Scholar. La búsqueda abarcó el periodo desde el 1/01/2001 a diciembre de 2016.

La estrategia de búsqueda consideró: Participation [title] AND (measuring [title/abstract] OR measure* [title/abstract] OR evaluation[title/abstract] OR assessment $^{*}$ [title/abstract]) AND (disability [title/abstract] OR disabilities[title/abstract] OR children [title/ abstract] OR child* [title/abstract]). Esta estrategia se adaptó a las distintas bases de datos utilizadas.

Los estudios que arrojó la búsqueda fueron seleccionados en forma independiente por dos investigadores (C.G. y A.M.), usando el software Abstrackr ${ }^{31}$ y las discrepancias se resolvieron por un tercero (P.N.). Los datos de los estudios fueron extraídos y analizados en Microsoft Excel. Para el análisis se utilizaron los datos de las validaciones originales de las escalas. Las validaciones transculturales de los instrumentos de evaluación no se consideraron en esta revisión.

\section{Resultados}

\section{Resultados de la búsqueda}

La búsqueda electrónica arrojó 1.689 artículos de los cuales, 28 cumplieron los criterios de inclusión, y a partir de éstos, se identificaron 9 escalas de evaluación de la participación en niños y/o adolescentes El detalle del proceso de selección se detalla en la figura 1 .

El detalle de los instrumentos seleccionados se desglosa en la tabla 2. Cabe mencionar que las escalas CAPE y PAC se consideraron como un solo instrumento ya que ambas escalas son complementarias entre sí y sus proceso de construcción y validación se realizó como un solo proceso ${ }^{32}$.

\section{Características de las escalas incluidas}

Las validaciones de 8 de los 9 instrumentos incluidos en esta revisión, fueron realizadas en población con y sin discapacidad (tabla 1). El estudio restante ${ }^{33}$ es declarado por los autores como un instrumento aplicable a la población con y sin discapacidad, pero los estudios de validación encontrados fueron realizados solo en niños(as) y adolescentes con discapacidad.

El rango etario considerado a nivel general va desde 


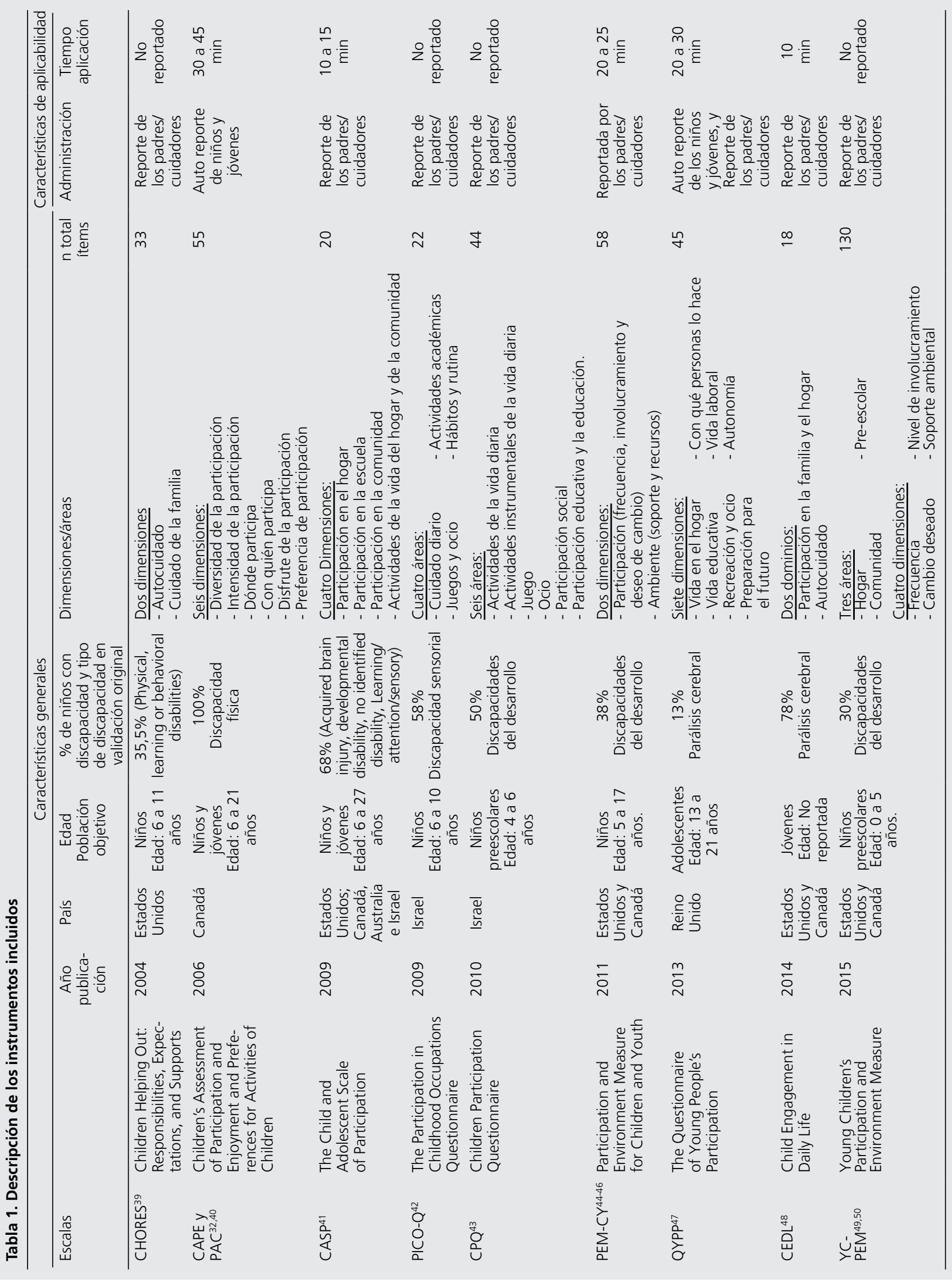




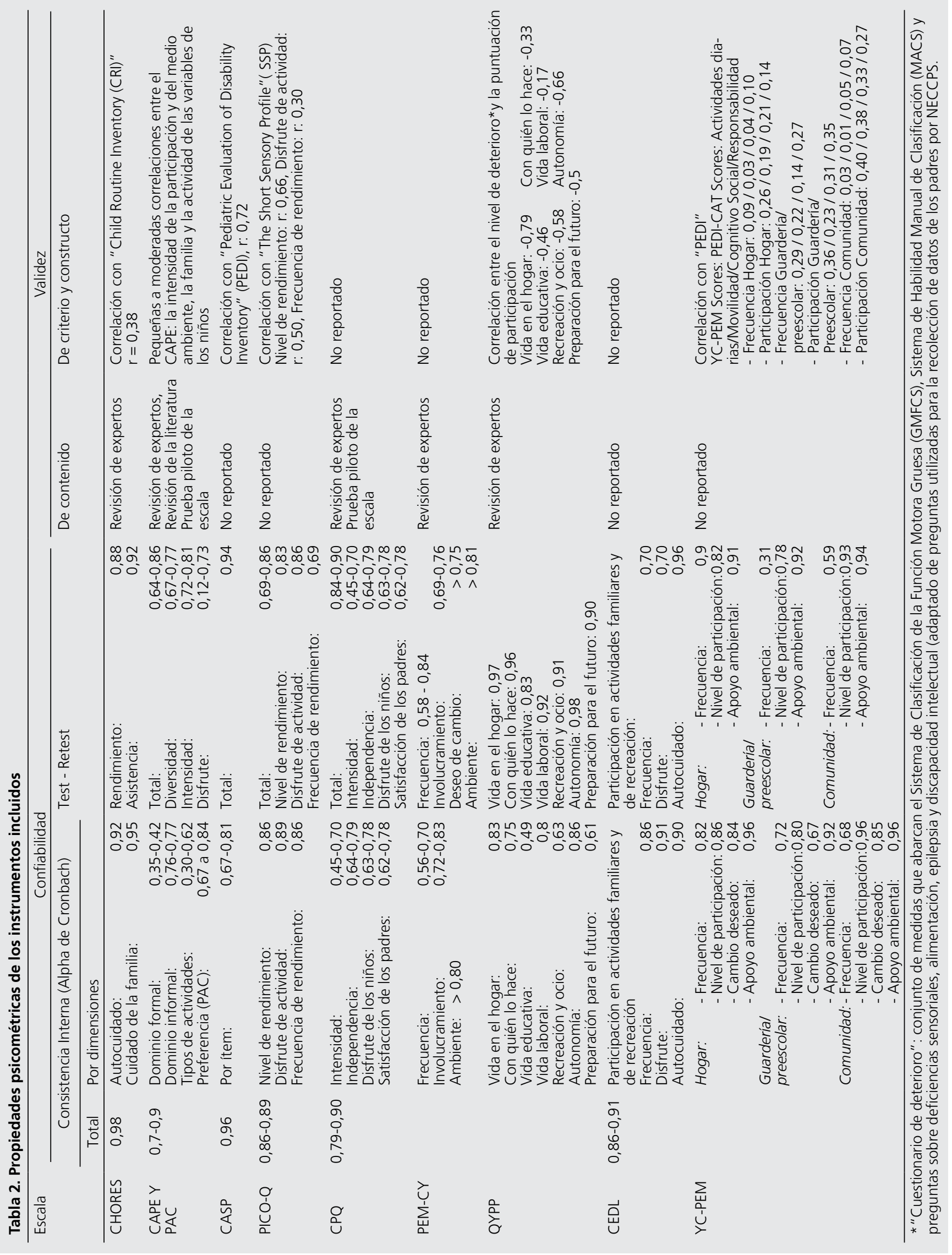


los 0 a los 27 años de edad. Dos de los instrumentos son para pre-escolares, tres para niños(as), uno para adolescentes, uno para jóvenes y dos para población infantil juvenil.

Siete de los 9 instrumentos son aplicados a padres y/o cuidadores, CAPE y PAC son de reporte de los(as) niños(as) y jóvenes y el QYPP es aplicable a niños(as) y sus padres/cuidadores. Los principales países en los que se han desarrollado estos instrumentos son Canadá, Estados Unidos, e Israel.

Los instrumentos se componen en promedio por 51 ítems, con un mínimo de 19 y un máximo de 197 ítems. Finalmente, respecto a la duración de su aplicación, esta varía desde los 5 a $45 \mathrm{~min}$. Todos los autores de los instrumentos incluidos declaran haberse basado en la definición de participación de la CIF.

\section{Propiedades psicométricas de los instrumentos incluidos}

Respecto de la validez (tabla 2), que se refiere a que la escala mide el concepto o constructo que pretende medir y se realiza a través de la validez de contenido, criterio y constructo ${ }^{34}$. Cuatro de los instrumentos no reportan la validez de contenido y los que sí lo hacen, lo realizan a través de paneles de expertos, considerando dentro de éstos a los padres, profesionales e investigadores, sólo la escala QYPP considera como expertos a los jóvenes con y sin discapacidad. La validez de criterio, es la propiedad psicométrica menos analizada, pues sólo dos escalas la estudian a través de correlaciones con otros instrumentos.

Respecto de las propiedades de confiabilidad (tabla 2) que se refiere a la reproducibilidad de la escala y se realiza tradicionalmente a través de $\alpha$ de Cronbach en el caso de la consistencia interna y a través de las pruebas test re-test ${ }^{34}$. Se evidencia que todas las escalas evalúan su consistencia interna, ya sea a nivel total o por cada una de sus dimensiones. La confiabilidad además, es evaluada por diez escalas a través del test-retest. Finalmente, los métodos inter e intra-evaluador en su mayoría no son realizados por los autores de las escalas y sólo dos de ellas reportan estas propiedades.

\section{Discusión}

La presente revisión sistemática contempló la revisión de 9 instrumentos que miden la participación en niños y adolescentes, siendo considerados aquellos que en su proceso de validación incorporaron niños, niñas y adolescentes con y sin discapacidad. Todos los autores de los instrumentos analizados declararon basarse en la definición de participación planteado por la CIF, sin embargo no se explica el detalle de cómo se realizó o si se incorporó en algún proceso de validación.

En relación a la validez de los instrumentos (tabla 2), es importante señalar que la validez de contenido es realizada a través de paneles de expertos, considerando dentro de éstos a los padres, profesionales e investigadores, sólo la escalas QYPP considera como expertos a los jóvenes. La Validez de criterio es la propiedad psicométrica menos analizada, pues sólo dos escalas la estudian a través de correlaciones con otros instrumentos. En cuanto a la validez de constructo, cinco escalas no reportan información y el mismo número da cuenta correlaciones con otras escalas.

Respecto de las propiedades de confiabilidad (tabla 2 ), se evidencia que todas las escalas evalúan su consistencia interna, ya sea a nivel total o por cada una de sus dimensiones. La confiabilidad además, es evaluada por diez escalas a través del test-retest. Finalmente, los métodos inter e intra-evaluador en su mayoría no son realizados por los autores de las escalas.

Ninguno de los instrumentos encontrados fue construido en países de habla hispana, sólo se encontró la traducción y la adaptación transcultural de las escalas CAPE Y PAC en Puerto Rico ${ }^{35}$ y España ${ }^{36}$. Además, se evidencia que en su mayoría, las escalas propuestas fueron construidas para la población con y sin discapacidad, no obstante, la mayoría de éstas fue construida para ser respondida por los padres y cuidadores, y no por los propios niños, niñas y jóvenes.

Lo anterior, sumado a que sólo una escala consideró como expertos en su construcción a los niños, niñas y jóvenes, resulta llamativo, pues al ser la participación el involucramiento en situaciones de la vida, resulta fundamental saber por parte de los propios participantes cómo evalúan su participación, por lo que se esperaría que más escalas se basaran en el auto-reporte de los niños, o en la complementariedad de éstos con reportes de sus padres o cuidadores.

Contar con instrumentos que puedan medir la participación de niños, niñas y adolescentes es fundamental para los distintos servicios que trabajan con ésta población, no sólo desde la rehabilitación, sino también desde la inclusión social. El poder comparar los resultados de la participación es fundamental para poder diseñar y ejecutar programas orientados a las necesidades particulares que se pudiesen detectar, entendiendo la participación no sólo como parte del desarrollo integral infantil y juvenil, sino también como un derecho ${ }^{37,38}$.

Además, para seguir profundizando y avanzando en la investigación en ésta área resulta imprescindible contar con instrumentos adecuados que permitan dar cuenta de la participación de niños y adolescentes. En especial debido a poder medir los distintos ámbitos que plantea la CIF.

Por otro lado, respecto de las potenciales limitaciones de esta revisión, se considera el no haber incluido 
instrumentos para poblaciones específicas (enfermedades crónicas) que también pudiesen ser aplicables a todo tipo de niños(as) y adolescentes. Debido a los criterios de inclusión, pudieron quedar fuera instrumentos cuyos resúmenes no se encontraban en español o inglés, o que cuyas validaciones psicométricas no estuviesen publicadas en la web, ya que no se realizó búsqueda manual.

La participación se considera fundamental para el desarrollo de todos los(as) niños(as) y adolescentes, siendo considerada uno de los ejes principales en el proceso de rehabilitación e inclusión de los niños, niñas y adolescentes con discapacidad. Por ello, la medición de la participación es uno de los desafíos más importantes incorporados posterior a la publicación de la CIF. Los distintos instrumentos incorporados en esta revisión sistemática dan cuenta de esto.

\section{Conclusiones}

Contar con instrumentos para evaluar la participación de niños(as) y adolescentes con y sin discapacidad se ha constituido como un desafío desde la publicación de la CIF en 2001. A nivel internacional se han construido diferentes escalas para evaluar este constructo, no obstante, en el contexto hispanoamericano sólo existen validaciones de las escalas CAPE Y PAC. Las escalas de participación, presentan una gran variedad respecto de sus dimensiones, número de ítems, formato de administración, entre otros aspectos evaluados por las escalas.

Si bien la construcción de estos instrumentos se basan lo propuesto por la CIF, éstas responden al constructo de participación existente en los países en que fueron diseñados (Canadá, Estados Unidos, Reino Unido), por lo que su aplicación en otros contextos, requiere de una adaptación transcultural y validación de propiedades psicométricas previo a su uso.

\section{Responsabilidades éticas}

Protección de personas y animales: Los autores declaran que los procedimientos seguidos se conformaron a las normas éticas del comité de experimentación humana responsable y de acuerdo con la Asociación Médica Mundial y la Declaración de Helsinki.

Confidencialidad de los datos: Los autores declaran que han seguido los protocolos de su centro de trabajo sobre la publicación de datos de pacientes.

Derecho a la privacidad y consentimiento informado: Los autores han obtenido el consentimiento informado de los pacientes y/o sujetos referidos en el artículo. Este documento obra en poder del autor de correspondencia.

\section{Conflicto de intereses}

Los autores declaran no tener conflicto de intereses.

\section{Referencias}

1. Organization WH. International classification of functioning, disability and health. Genova, Suiza: WHO; 2001.

2. Rimmer JH. Use of the ICF in identifying factors that impact participation in physical activity/rehabilitation among people with disabilities. Disability and rehabilitation. 2006;28(17):1087-95.

3. Hoogsteen L, Woodgate RL. Can I Play? A Concept Analysis of Participation in Children with Disabilities. Physical \& occupational therapy in pediatrics. 2010;30(4):325-39.

4. Piskur B, Daniels R, Jongmans MJ, et al Participation and social participation: are they distinct concepts? Clin Rehabil. 2014;28(3):211-20.

5. Law M, Finkelman S, Hurley P, et al. Participation of children with physical disabilities: relationships with diagnosis, physical function, and demographic variables. Scandinavian Journal of Occupational Therapy. 2004;11(4):15662.

6. Law M. Participation in the occupations of everyday life. The American journal of occupational therapy: official publication of the American Occupational Therapy Association. 2002;56(6):640-9.

7. Shikako-Thomas K, Shevell M, Schmitz $\mathrm{N}$, et al. Determinants of participation in leisure activities among adolescents with cerebral palsy. Research in developmental disabilities. 2013;34(9):2621-34.

8. Law M, King G, King S, et al. Patterns of participation in recreational and leisure activities among children with complex physical disabilities. Developmental medicine and child neurology. 2006;48(5):337-42.

9. Coleman D, Iso-Ahola SE. Leisure and health: The role of social support and selfdetermination. Journal of leisure research. 1993;25(2):111.

10. Shikako-Thomas K, Dahan-Oliel N,
Shevell M, et al. Play and be happy? Leisure participation and quality of life in school-aged children with cerebral palsy. International journal of pediatrics. 2012;2012:387280.

11. Odom SL, Buysse V, Soukakou E. Inclusion for young children with disabilities: A quarter century of research perspectives. Journal of Early Intervention. 2011;33(4):344-56.

12. Kinney W, Coyle C. Predicting life satisfaction among adults with physical disabilities. Archives of physical medicine and rehabilitation. 1992;73(9):863-9.

13. Giaconi C, Nahuelhual P, Pedrero V. Participación de niños y adolescentes en situación de discapacidad: un ámbito a ser estudiado y evaluado. Revista Chilena de Pediatría. 2016.

14. Goldstein DN, Cohn E, Coster W. Enhancing participation for children with disabilities: application of the ICF enablement framework to pediatric 
physical therapist practice. Pediatric physical therapy : the official publication of the Section on Pediatrics of the American Physical Therapy Association. 2004;16(2):114-20.

15. Schreuer N, Sachs D, Rosenblum S. Participation in leisure activities: Differences between children with and without physical disabilities. Research in developmental disabilities. 2014;35(1):223-33.

16. Bedell G, Coster W. Measuring participation of school-aged children with traumatic brain injuries: considerations and approaches. J Head Trauma Rehabil. 2008;23(4):220-9.

17. Coster W, Khetani M. Measuring participation of children with disabilities: Issues and challenges. Disability \& Rehabilitation. 2008.

18. Phillips RL, Olds T, Boshoff K, Lane AE. Measuring activity and participation in children and adolescents with disabilities: A literature review of available instruments. Australian occupational therapy journal. 2013;60(4):288-300.

19. Morris C. Measuring children's participation. Developmental medicine and child neurology. 2007;49(9):645.

20. Perenboom RJM, Chorus AMJ. Measuring participation according to the International Classification of Functioning, Disability and Health (ICF). Disability \& Rehabilitation. 2003;25(11/12):577-87.

21. Taylor R. Measuring the activity and participation of children with and without disabilities in everyday tasks. 2011.

22. Pollard B, Dixon D, Dieppe P, Johnston M. Measuring the ICF components of impairment, activity limitation and participation restriction: an item analysis using classical test theory and item response theory. Health Qual Life Outcomes. 2009;7:41.

23. Sakzewski L, Boyd R, Ziviani J. Clinimetric properties of participation measures for 5- to 13-year-old children with cerebral palsy: a systematic review. Developmental medicine and child neurology. 2007;49(3):232-40.

24. Chien CW, Rodger S, Copley J, McLaren C. Measures of participation outcomes related to hand use for 2- to 12-year-old children with disabilities: a systematic review. Child Care Health Dev. 2014;40(4):458-71.

25. Magasi S, Post MW. A Comparative Review of Contemporary Participation Measures' Psychometric Properties and Content Coverage. Archives of Physical Medicine \& Rehabilitation. 2010;91(9):S17-28.
26. Schoneveld K, Wittink H, Takken T. Clinimetric evaluation of measurement tools used in hand therapy to assess activity and participation. Journal of Hand Therapy. 2009;22(3):221.

27. Reitsma JBRA, Whiting P, Vlassov VV, Leeflang MMG, Deeks JJ. Assessing methodological quality. Cochrane Handbook for Systematic Reviews of Diagnostic Test Accuracy Version 100: The Cochrane Collaboration; 2009.

28. Moher D, Liberati A, Tetzlaff J, Altman DG. Preferred reporting items for systematic reviews and meta-analyses: the PRISMA statement. Annals of internal medicine. 2009;151(4):264-9.

29. Nahuelhual PGC, Machuca A. Measuring participation in children and adolescents with and without disabilities: a systematic review. Prospero. 2015;CRD42015020644.

30. Sakzewski L, Boyd R, Ziviani J. Clinimetric properties of participation measures for 5- to 13-year-old children with cerebral palsy: a systematic review. Developmental Medicine \& Child Neurology. 2007;49(3):232-40.

31. Wallace BC, Small K, Brodley CE, Lau J, Trikalinos TA, editors. Deploying an interactive machine learning system in an evidence-based practice center: abstrackr. Proceedings of the 2nd ACM SIGHIT International Health Informatics Symposium; 2012: ACM.

32. King GA, Law M, King S, et al. Measuring children's participation in recreation and leisure activities: construct validation of the CAPE and PAC. Child Care Health Dev. 2007;33(1):28-39.

33. King G. CAPE/PAC manual: Children's assessment of participation and enjoyment \& preferences for activities of children. 2004.

34. Streiner DL, Norman GR, Cairney J. Health measurement scales: a practical guide to their development and use: Oxford University Press, USA; 2015.

35. Colón WI, Rodríguez C, Ito M, Reed CN. Psychometric evaluation of the Spanish version of the Children's Assessment of Participation and Enjoyment and Preferences for Activities of Children. Occupational therapy international. 2008;15(2):100-13

36. Longo E, Badia M, Orgaz B, Verdugo MA. Cross-cultural validation of the Children's Assessment of Participation and Enjoyment (CAPE) in Spain. Child: care, health and development. 2014;40(2):23141.

37. UNICEF. State of the World's Children: Celebrating 20 Years of the Convention on the Rights of the Child: Unicef; 2009.

38. UNICEF. Guidelines for Measuring the Participation of Children and Adolescents. 2007.

39. Dunn L. Validation of the CHORES: a measure of school-aged children's participation in household tasks. Scandinavian Journal of Occupational Therapy. 2004.

40. King G, Law M, King S. Children's Assessment of Participation and Enjoyment and Preferences for Activities of Children (CAPE/PAC) manual. 2004.

41. Bedell G. Further validation of the Child and Adolescent Scale of Participation (CASP). Dev Neurorehabil. 2009;12(5):342-51.

42. Bar-Shalita T, Yochman A, ShapiroRihtman T, Vatine J, Parush S. The Participation in Childhood Occupations Questionnaire (PICO-Q): a pilot study. Physical \& occupational therapy in pediatrics. 2009;29(3):295-310.

43. Rosenberg L, Jarus T, Bart O. Development and initial validation of the Children Participation Questionnaire (CPQ). Disabil Rehabil. 2010;32(20):1633-44.

44. Coster W, Bedell G, Law M, et al. Psychometric evaluation of the Participation and Environment Measure for Children and Youth. Developmental medicine and child neurology. 2011;53(11):1030-7.

45. Coster W, Law M. The Participation and Environment Measure for Children and Youth (PEM- CY): An innovative measure for home, school and community. ... for Disability .... 2013.

46. Coster W, Law M, Bedell G. Participation and environment measure for children and youth (PEM-CY). Boston (MA): Boston .... 2010.

47. Tuffrey C, Bateman BJ, Colver AC. The Questionnaire of Young People's Participation (QYPP): a new measure of participation frequency for disabled young people. Child: care, health and development. 2013;39(4):500-11.

48. Chiarello LA, Palisano RJ, McCoy SW, et al. Child engagement in daily life: a measure of participation for young children with cerebral palsy. Disabil Rehabil. 2014;36(21):1804-16.

49. Khetani MA. Validation of Environmental Content in the Young Children's Participation and Environment Measure. Archives of Physical Medicine \& Rehabilitation. 2015;96(2):317-22.

50. Khetani MA, Graham JE, Davies PL, Law MC, Simeonsson RJ. Psychometric Properties of the Young Children's Participation and Environment Measure. Archives of Physical Medicine \& Rehabilitation. 2015;96(2):307-16. 